Article

\title{
Fine-Tuning Approach for Segmentation of Gliomas in Brain Magnetic Resonance Images with a Machine Learning Method to Normalize Image Differences among Facilities
}

\author{
Satoshi Takahashi ${ }^{1,2,+}$, Masamichi Takahashi ${ }^{3,4, *,+} \oplus$, Manabu Kinoshita ${ }^{5}{ }^{9}$, Mototaka Miyake ${ }^{6}$, \\ Risa Kawaguchi ${ }^{7}$, , Naoki Shinojima ${ }^{8}$, Akitake Mukasa ${ }^{8}$, Kuniaki Saito ${ }^{9}$, Motoo Nagane ${ }^{9}$, Ryohei Otani ${ }^{10,11}$, \\ Fumi Higuchi ${ }^{10}$, Shota Tanaka ${ }^{12}$, Nobuhiro Hata ${ }^{13}{ }^{1}$, Kaoru Tamura ${ }^{14}$, Kensuke Tateishi ${ }^{15}$, Ryo Nishikawa ${ }^{16}$, \\ Hideyuki Arita ${ }^{5}$, Masahiro Nonaka ${ }^{17,18}$, Takehiro Uda ${ }^{19} \mathbb{D}$, Junya Fukai ${ }^{20}{ }^{\circ}$, Yoshiko Okita ${ }^{18,21}$, \\ Naohiro Tsuyuguchi ${ }^{19,22}$, Yonehiro Kanemura ${ }^{18,23}$, Kazuma Kobayashi ${ }^{1,2}$, Jun Sese ${ }^{7,24}$, Koichi Ichimura ${ }^{4}{ }^{(1)}$, \\ Yoshitaka Narita ${ }^{3}$ and Ryuji Hamamoto ${ }^{1,2}$
}

check for

updates

Citation: Takahashi, S.; Takahashi,

M.; Kinoshita, M.; Miyake, M.;

Kawaguchi, R.; Shinojima, N.;

Mukasa, A.; Saito, K.; Nagane, M.;

Otani, R.; et al. Fine-Tuning Approach

for Segmentation of Gliomas in Brain

Magnetic Resonance Images with a

Machine Learning Method to

Normalize Image Differences among

Facilities. Cancers 2021, 13, 1415.

https://doi.org/10.3390/

cancers13061415

Academic Editor: Jorn Fierstra

Received: 27 December 2020

Accepted: 16 March 2021

Published: 19 March 2021

Publisher's Note: MDPI stays neutral with regard to jurisdictional claims in published maps and institutional affiliations.

Copyright: (c) 2021 by the authors. Licensee MDPI, Basel, Switzerland. This article is an open access article distributed under the terms and conditions of the Creative Commons Attribution (CC BY) license (https:// creativecommons.org/licenses/by/ $4.0 /$ )
1 Division of Molecular Modification and Cancer Biology, National Cancer Center Research Institute, 5-1-1 Tsukiji, Chuo-ku, Tokyo 104-0045, Japan; satoshi.takahashi.fy@riken.jp (S.T.);

kazumkob@ncc.go.jp (K.K.); rhamamot@ncc.go.jp (R.H.)

2 Cancer Translational Research Team, RIKEN Center for Advanced Intelligence Project, 1-4-1 Nihonbashi, Chuo-ku, Tokyo 103-0027, Japan

3 Department of Neurosurgery and Neuro-Oncology, National Cancer Center Hospital, 5-1-1 Tsukiji, Chuo-ku, Tokyo 104-0045, Japan; yonarita@ncc.go.jp

4 Division of Brain Tumor Translational Research, National Cancer Center Research Institute, 5-1-1 Tsukiji, Chuo-ku, Tokyo 104-0045, Japan; kichimur@ncc.go.jp

5 Department of Neurosurgery, Osaka University Graduate School of Medicine, 2-2 Yamadaoka, Suita, Osaka 565-0871, Japan; mail@manabukinoshita.com (M.K.); h-arita@nsurg.med.osaka-u.ac.jp (H.A.)

6 Department of Diagnostic Radiology, National Cancer Center Hospital, 5-1-1 Tsukiji, Chuo-ku, Tokyo 104-0045, Japan; mmiyake@ncc.go.jp

7 Artificial Intelligence Research Center, National Institute of Advanced Industrial Science and Technology, 2-3-26, Aomi, Koto-ku, Tokyo 135-0064, Japan; rkawaguc@cshl.edu (R.K.); sesejun@gmail.com (J.S.)

8 Department of Neurosurgery, Graduate School of Medical Sciences, Kumamoto University, 1-1-1 Honjo, Chuo-ku, Kumamoto 860-8556, Japan; nshinojima@kuh.kumamoto-u.ac.jp (N.S.); mukasa@kumamoto-u.ac.jp (A.M.)

9 Department of Neurosurgery, Kyorin University Faculty of Medicine, 6-20-2, Sinkawa, Mitaka, Tokyo 181-8611, Japan; kusaito-tky@umin.ac.jp (K.S.); mnagane@ks.kyorin-u.ac.jp (M.N.)

10 Department of Neurosurgery, Dokkyo Medical University, 880 Kitakobayashi, Mibu, Shimotsugagun, Tochigi 321-0293, Japan; ryouhei-ohtani@umin.ac.jp (R.O.); fhiguchi@dokkyomed.ac.jp (F.H.)

11 Department of Neurosurgery, Tokyo Metropolitan Komagome Hospital, 3-18-22 Honkomagome, Bunkyo-ku, Tokyo 113-8677, Japan

12 Department of Neurosurgery, Faculty of Medicine, The University of Tokyo, 7-3-1 Hongo Bunkyo-ku, Tokyo 113-8655, Japan; tanakas-tky@umin.ac.jp

13 Department of Neurosurgery, Graduate School of Medical Sciences, Kyushu University, 3-1-1 Maidashi, Higashi-ku, Fukuoka 812-8582, Japan; hatanobu@ns.med.kyushu-u.ac.jp

14 Department of Neurosurgery, Tokyo Medical and Dental University, 1-5-45 Yushima, Bunkyo-ku, Tokyo 113-8510, Japan; tamura.nsrg@tmd.ac.jp

15 Department of Neurosurgery, Graduate School of Medicine, Yokohama City University, 3-9 Fukuura, Kanazawa-ku, Yokohama, Kanagawa 236-0004, Japan; ktate12@yokohama-cu.ac.jp

16 Department of Neuro-Oncology/Neurosurgery, Saitama Medical University International Medical Center, 397-1 Yamane, Hidaka, Saitama 350-1298, Japan; rnishika@saitama-med.ac.jp

17 Department of Neurosurgery, Kansai Medical University, 2-5-1 Shinmachi, Hirakata, Osaka 573-1010, Japan; nonakamasa65@gmail.com

18 Department of Neurosurgery, National Hospital Organization Osaka National Hospital, 2-1-14 Hoenzaka, Chuo-ku, Osaka 540-0006, Japan; yokita4246@gmail.com (Y.O.); yonehirok@gmail.com (Y.K.)

19 Department of Neurosurgery, Osaka City University Graduate School of Medicine, 1-4-3 Asahi-machi, Abeno-ku, Osaka 545-8585, Japan; uda@med.osaka-cu.ac.jp (T.U.); ntsuyuguchi@gmail.com (N.T.)

20 Department of Neurological Surgery, Wakayama Medical University School of Medicine Wakayama, 811-1 Kimiidera, Wakayama 641-8509, Japan; junfukai@wakayama-med.ac.jp

21 Department of Neurosurgery, Osaka International Cancer Institute, 3-1-69 Ootemae, Chuo-ku, Osaka 541-8567, Japan

22 Department of Neurosurgery, Kindai University Faculty of Medicine, 377-2 Ohnohigashi, Osaka-Sayama, Osaka 589-8511, Japan 
23 Department of Biomedical Research and Innovation, Institute for Clinical Research, National Hospital Organization Osaka National Hospital, 2-1-14 Hoenzaka, Chuo-ku, Osaka 540-0006, Japan

24 Humanome Lab, 2-4-10 Tsukiji, Chuo-ku, Tokyo 104-0045, Japan

* Correspondence: masataka@ncc.go.jp; Tel.: +81-3-3542-2511; Fax: +81-3-3542-2551

+ These authors contributed equally to this work.

Simple Summary: This study evaluates the performance degradation of machine learning models for segmenting gliomas in brain magnetic resonance images caused by domain shift and proposed possible solutions. Although machine learning models exhibit significant potential for clinical applications, performance degradation in different cohorts is a problem that must be solved. In this study, we identify the impact of the performance degradation of machine learning models to be significant enough to render clinical applications difficult. This demonstrates that it can be improved by fine-tuning methods with a small number of cases from each facility, although the data obtained appeared to be biased. Our method creates a facility-specific machine learning model from a small real-world dataset and public dataset; therefore, our fine-tuning method could be a practical solution in situations where only a small dataset is available.

Abstract: Machine learning models for automated magnetic resonance image segmentation may be useful in aiding glioma detection. However, the image differences among facilities cause performance degradation and impede detection. This study proposes a method to solve this issue. We used the data from the Multimodal Brain Tumor Image Segmentation Benchmark (BraTS) and the Japanese cohort (JC) datasets. Three models for tumor segmentation are developed. In our methodology, the BraTS and JC models are trained on the BraTS and JC datasets, respectively, whereas the fine-tuning models are developed from the BraTS model and fine-tuned using the JC dataset. Our results show that the Dice coefficient score of the JC model for the test portion of the JC dataset was $0.779 \pm 0.137$, whereas that of the BraTS model was lower $(0.717 \pm 0.207)$. The mean Dice coefficient score of the fine-tuning model was $0.769 \pm 0.138$. There was a significant difference between the BraTS and JC models $(p<0.0001)$ and the BraTS and fine-tuning models $(p=0.002)$; however, no significant difference between the JC and fine-tuning models $(p=0.673)$. As our fine-tuning method requires fewer than 20 cases, this method is useful even in a facility where the number of glioma cases is small.

Keywords: glioma; machine learning; MR images; fine-tuning; deep learning

\section{Introduction}

Glioma is the most frequent primary intracerebral tumor in adults. The prognosis for most glioma patients is fatal, and the disease has a significant impact on the physical, psychological, and social status of the patients and their families [1]. The World Health Organization (WHO) grade of glioma represents its biological behavior and correlates with the number of accumulated gene mutations [2,3]. Although the treatment strategy differs with the type of glioma and WHO grade, a combination of surgical resection, radiotherapy, and chemotherapy is generally applied [4-7]. Because of the invasive nature of gliomas, it is difficult to remove all tumor cells within an adequate safety margin; however, the prognosis of patients with glioma improves as the extent of resection increases [8]. Therefore, it is valuable to identify tumor boundaries before surgery.

Magnetic resonance (MR) imaging is routinely used to diagnose and determine treatment strategies for gliomas. Compared with computed tomography, MR imaging has the advantage of providing more detailed information regarding tumors and surrounding tissues. Therefore, MR imaging is preferably used to (1) determine the extent of a tumor by neuroradiologists, (2) monitor the boundary of lesions when neurosurgeons perform glioma resection with a navigation system, and (3) evaluate the effect of treatment by neurooncologists. However, manual segmentation of brain tumors from numerous MR images generated in clinical routine is a time-consuming and challenging task. Hence, 
there is a need for a technology that allows automated brain tumor segmentation. It is an urgent mission to build a machine learning model for the segmentation of brain tumors.

The most notable network architecture in machine learning for segmenting tumors is U-Net [9], and several improved models have already been developed [10-15]. For example, Myronenko won the first prize in the Brain Tumor Segmentation Competition (BraTs) in 2018, with a very high Dice coefficient score $(\geq 0.90)$ [16,17].

Despite the current rapid progress in using machine learning and deep learning technologies in the medical field [18-26], the clinical application of machine learning models for tumor segmentation still requires a significant amount of progress. One reason for the delay in its clinical application appears to be the performance degradation caused by domain shift [27]. Most machine learning models for the segmentation of MR images in recent years use deep learning techniques. The aforementioned U-Net is also an example of deep learning architecture. When training these architectures, one often assumes that the training data and test data are sampled from the same distribution [28]. In medical imaging, images obtained from one facility (one imaging device and one image protocol) can be assumed as a part of one domain. Therefore, when a deep learning model is created from data acquired from facility A (domain A), the model can display high prediction accuracy for newly acquired data from domain A. However, suppose the model is applied to data from another facility, B (domain B). In that case, the performance may drop significantly if the data distributions of domains A and B are different (this is called 'domain shift'). Thus, the clinical application of deep learning models for segmenting MR images has not been established. The performance degradation, due to the domain shift, is a critical issue that must be handled in a real clinical setting because the impact and frequency of the performance degradation caused by domain shift are unpredictable. To understand the mechanisms involved in performance degradation, due to the domain shift, we must carefully analyze data from multiple facilities and multiple domains.

In this study, we aimed (1) to clarify the detailed mechanisms of performance degradation caused by a domain shift by collecting images from 10 different facilities in Japan, and (2) to develop a method to solve this issue.

\section{Materials and Methods}

\subsection{Ethics}

This study was approved by the Ethics Committees of all the facilities that provided the images (IRB number: 2013-042) the Ethics Committee of the National Cancer Center, Tokyo, Japan (approval ID: 2016-496). All methods were performed in accordance with the Ethical Guidelines for Medical and Health Research Involving Human Subjects.

\subsection{JC Dataset}

Preoperative (before surgical resection or biopsy) MR images were tried to be collected from 951 adult diffuse glioma patients from 10 facilities in Japan between November 1991 and October 2015; the patients had participated in our previous study [29] in which we demonstrated that the combination of IDH mutation status, TERT promoter mutation status, and MGMT (O-6-methylguanine-DNA methyltransferase) methylation status refined the classification of WHO grade II-IV gliomas. Of the 951 cases, 673 cases had preoperative digital MR images in at least one sequence, and 544 cases met our criteria described below. Clinical information was collected in detail, and sequencing analysis was performed on the tumor specimens collected from these patients. We named these images the Japanese cohort (JC) dataset. The images satisfying the following three categories were selected:

1. All four types of images, T1-weighted images (T1), T2-weighted images (T2), fluidattenuated inversion recovery (FLAIR), and T1-weighted images with gadolinium enhancement (GdT1), were eligible.

2. Surgical removal or biopsy was performed. 
3. Diagnostic tests, including genetic analysis of key biomarkers (IDH mutation and 1p19q), were performed following the WHO 2007 or 2016 classifications of Central Nerves System tumors.

We further divided 544 subjects into three categories, as described above. The number of patients included in the JC dataset was 1.6-times larger than that in the Multimodal Brain Tumor Image Segmentation Benchmark (BraTS) dataset. To the best of our knowledge, this is the largest glioma image dataset in which genetic/epigenetic profiles and clinical information are accessible. Table 1 presents detailed information on the clinical characteristics of the JC dataset.

Table 1. Clinical characteristics of the cases in this study.

\begin{tabular}{|c|c|c|c|c|c|c|c|c|c|c|c|}
\hline Parameter & $\begin{array}{c}\text { All } \\
\text { Dataset }\end{array}$ & $\begin{array}{c}\text { Facility } \\
\text { A }\end{array}$ & $\begin{array}{c}\text { Facility } \\
\text { B }\end{array}$ & $\begin{array}{c}\text { Facility } \\
\text { C }\end{array}$ & $\begin{array}{c}\text { Facility } \\
\text { D }\end{array}$ & $\begin{array}{c}\text { Facility } \\
\text { E }\end{array}$ & $\begin{array}{c}\text { Facility } \\
\text { F }\end{array}$ & $\begin{array}{l}\text { Facility } \\
\text { G }\end{array}$ & $\begin{array}{c}\text { Facility } \\
\text { H }\end{array}$ & $\begin{array}{c}\text { Facility } \\
\text { I }\end{array}$ & $\begin{array}{c}\text { Facility } \\
\mathrm{J}\end{array}$ \\
\hline $\begin{array}{l}\text { Median age } \\
\text { (range) }\end{array}$ & $\begin{array}{c}60 \\
(86- \\
17)\end{array}$ & $\begin{array}{c}54 \\
(81- \\
28)\end{array}$ & $\begin{array}{c}64.5 \\
(84- \\
26)\end{array}$ & $\begin{array}{l}64.5 \\
(85- \\
25)\end{array}$ & $\begin{array}{c}66 \\
(85- \\
51)\end{array}$ & $\begin{array}{c}59 \\
(80- \\
25)\end{array}$ & $\begin{array}{c}57 \\
(86- \\
17)\end{array}$ & $\begin{array}{c}60 \\
(79- \\
22)\end{array}$ & $\begin{array}{l}55.5 \\
(80- \\
21)\end{array}$ & $\begin{array}{c}54 \\
(76- \\
28)\end{array}$ & $\begin{array}{c}61 \\
(81- \\
19)\end{array}$ \\
\hline \multicolumn{12}{|l|}{ Sex } \\
\hline Male & 293 & 92 & 20 & 50 & 8 & 32 & 17 & 21 & 23 & 16 & 23 \\
\hline Female & 251 & 65 & 20 & 44 & 5 & 27 & 14 & 21 & 21 & 13 & 12 \\
\hline \multicolumn{12}{|l|}{ LrGG or GBM } \\
\hline LrGG & 218 & 71 & 18 & 0 & 0 & 31 & 18 & 25 & 23 & 18 & 14 \\
\hline GBM & 326 & 86 & 22 & 94 & 13 & 28 & 13 & 17 & 21 & 11 & 21 \\
\hline \multicolumn{12}{|l|}{ WHO grade } \\
\hline II & 91 & 34 & 12 & 0 & 0 & 7 & 5 & 10 & 10 & 7 & 6 \\
\hline III & 127 & 37 & 6 & 0 & 0 & 24 & 13 & 15 & 13 & 11 & 8 \\
\hline IV & 326 & 86 & 22 & 94 & 13 & 28 & 13 & 17 & 21 & 11 & 21 \\
\hline \multicolumn{12}{|l|}{ Pathological diagnosis } \\
\hline Diffuse astrocytoma & 66 & 27 & 9 & 0 & 0 & 4 & 3 & 9 & 7 & 4 & 3 \\
\hline Anaplastic astrocytoma & 88 & 25 & 3 & 0 & 0 & 16 & 6 & 14 & 10 & 7 & 7 \\
\hline Oligodendroglioma & 25 & 7 & 3 & 0 & 0 & 3 & 2 & 1 & 3 & 3 & 3 \\
\hline Anaplastic oligodendroglioma & 39 & 12 & 3 & 0 & 0 & 8 & 7 & 1 & 3 & 4 & 1 \\
\hline Glioblastoma & 326 & 86 & 22 & 94 & 13 & 28 & 13 & 17 & 21 & 11 & 21 \\
\hline
\end{tabular}

LrGG = lower grade glioma, GBM = glioblastoma.

\subsection{BraTS Dataset}

We also used the BraTS 2019 dataset as the BraTS dataset, including T1, T2, FLAIR, and GdT1 images of 259 high-grade glioma cases and 76 low-grade glioma cases with tumor volume of interest (VOI) information [16,30,31]. Further details regarding the BraTS dataset can be found elsewhere [16,30].

\subsection{Creating VOI}

All VOIs in our JC dataset were manually created by skilled neuroradiologists using in-house tools. As shown in Supplementary Figure S1, the VOIs were created based on the information regarding four sequences. The VOI was defined as an area that may have a tumor, including edema. We included the T2 hyper lesions in the VOI because they may contain non-enhancing tumor lesions. We think that the advantages of including non-enhancing tumor outweigh the disadvantages of including 'pure edema' that may make noise. The created VOI included parts referred to as 'necrotic tumor core'.

In contrast, the VOI in the BraTS datasets was classified into three parts that included 'edema', 'tumor core', and 'necrotic tumor core'. Edema, tumor core, and necrotic tumor core were merged to transfer the information and optimize the VOI of the JC dataset, and was, therefore, redefined as the new VOI of the BraTS dataset.

\subsection{Image Preparation}

The images from the BraTS dataset were cropped and resized to $176 \times 192 \times 160$, using a script written in Python 3.7.7 (https:/ / www.python.org, accessed on 14 October 2020). 
The images from the JC dataset were skull stripped by BET [32]. Then, the skull stripped images were cropped and resized to $176 \times 192 \times 160$ using our Python script.

\subsection{Machine Learning Model}

First, the JC dataset was randomly divided into two halves; one was used for training and fine-tuning ('JC dataset pre-training'; Figure 1), while the other was an independent test dataset. To optimize the distribution of the cases, we ensured that both training and test datasets have approximately the same number of images from each facility. Second, three types of machine learning models were created for segmentation. Finally, the performances of these models on the independent test dataset were evaluated using the Dice coefficient score. Figure 1 shows the overall flow.

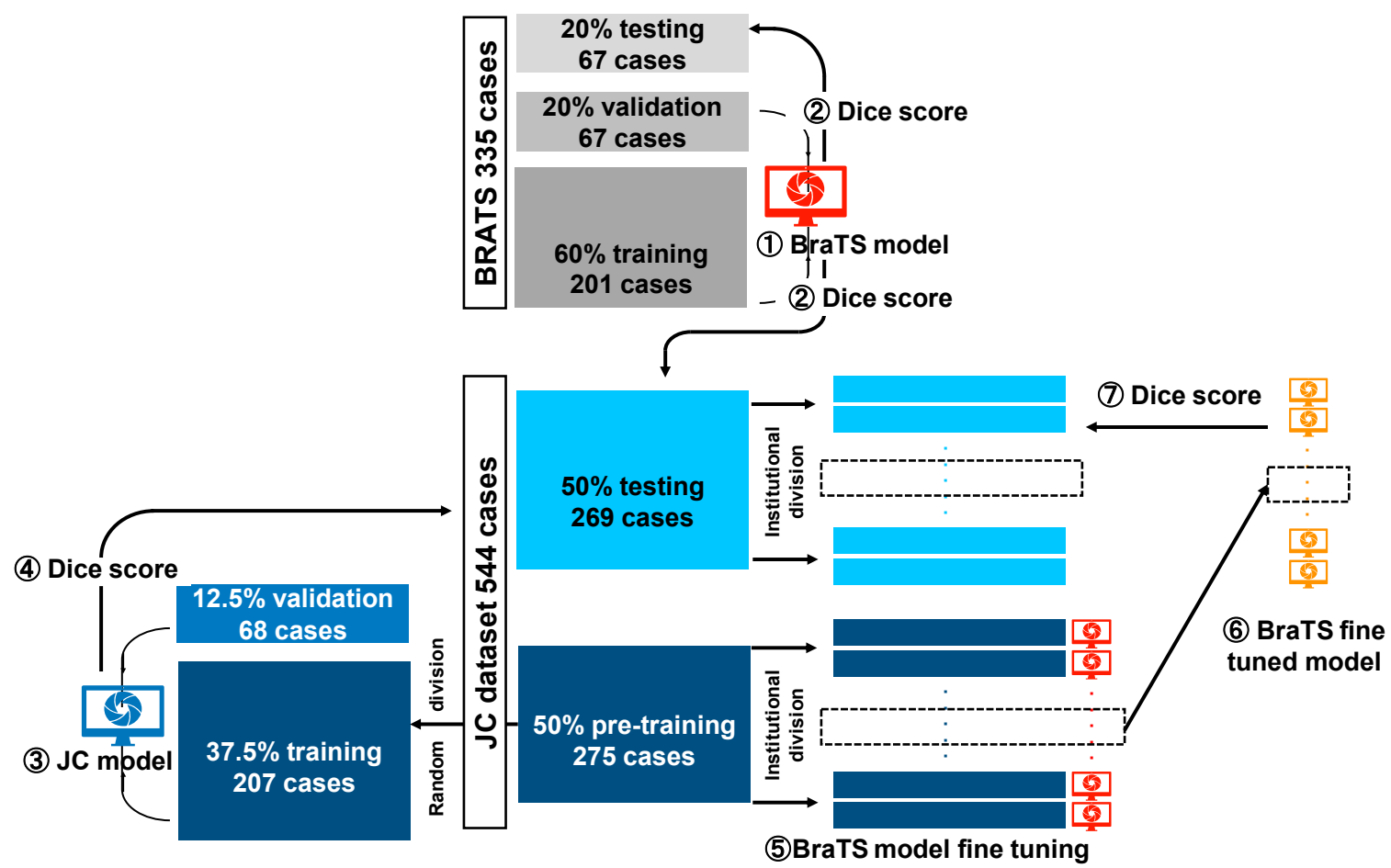

Figure 1. The overall flow of this research. Three types of machine learning models were built for segmentation: The Multimodal Brain Tumor Image Segmentation Benchmark (BraTS) model, the Japanese cohort (JC) model, and the finetuning model. (1) The BraTS model was trained in the training part of the BraTS dataset. (2) The BraTS model was evaluated by the Dice coefficient scores of the BraTS data set and the test portion of the JC data set. (3) The JC model was trained on the training portion of the JC data set. (4) The JC model was evaluated by the Dice coefficient score of the test portion of the JC data set. (5) The BraTS model was fine-tuned for optimal analysis at each facility. (6) The BraTS model fine-tuned by the aforementioned method, was named the fine-tuning model. (7) The fine-tuning model was evaluated with the Dice coefficient score of the test portion of the JC data set.

1. BraTS model: The BraTS dataset was split into training, validation, and test sets (60\% training, $20 \%$ validation, and $20 \%$ test), and the model was trained for tumor segmentation on the test set.

2. JC model: The pre-training part of the JC dataset was further split into training and validation sets ( $75 \%$ training and $25 \%$ validation), and the model was trained on the training portion of the JC dataset.

3. Fine-tuning model: The BraTS model was fine-tuned to perform an optimized analysis in each facility. A maximum of 20 cases (randomly selected) from the pre-training portion of the JC dataset were used for fine-tuning. Here, if a facility had fewer than 20 training cases, all the training portions in the JC dataset from the facility were used for fine-tuning. 
The architecture of our machine learning model (Supplementary Figure S2 and Table S1) is based on the method described elsewhere [9,33,34]. As shown in Supplementary Figure S2, we grouped all layers between two element-wise sums or merged operation layers into one block for further technical convenience. Most blocks contain three 3D convolution layers and one dropout layer. The concept of each group corresponds to the blue shaded areas in Supplementary Figure S2. Each block was named in order from the shallowest to the deepest block as '1st down', '2nd down', ... '5th down' and then from the deepest to the shallowest block as '1st up', '2nd up', ... '5th up'. The optimizer was set to AdaGrad, and the learning rate was set to 0.005 . The deep learning models were developed using the Keras package in Python (https:/ / keras.io, accessed on 14 October 2020). The network architectures for all three models were identical. RMSprop was used as the optimizer, and the learning rate was set to 0.0005. All the images were cropped and input to the model for segmentation as a 4ch (T1, T2, FLAIR, GdT1) 3D volume of $4 \times 176 \times 192 \times 160$.

\subsection{Finding the Best Fine-Tuning Method}

The fine-tuning model is specialized for each facility; therefore, the number of the finetuning model is the same as that of the facilities. To determine the most appropriate finetuning method, we decided to use the training data of the JC dataset from facility A because facility A had a relatively large dataset (157 cases). Twenty cases were randomly selected from the training data. The remaining training data were used for performance evaluation.

Initially, we examined three different methods: (1) The first method was to train a model in which all layers were layers with learnable parameters (learnable layers; 'fine_all' model); (2) the second method was to train a model in which only the down path was a learnable layer ('down model'; in other words, a model in which the up path was frozen); and (3) the third method was to train a model in which only the up path was capable of learning ('up model'; Supplementary Figure S3).

Next, we investigated the effect of horizontal connection instead of the vertical connections of 3D U-Net on fine-tuning (Supplementary Figure S4). In this experiment, we re-examined three different methods: (1) The first method was to train a model in which all layers were learnable layers as mentioned above ('fine_all' model), (2) the second method was to train a model in which learnable layers were those in the 1st down and 1st up blocks ('down1_up1 model'), and (3) the third method was to train a model in which learnable layers were those in the 1st down, 2nd down, 1st up, and 2nd up blocks ('down2_up2 model'). The fine-tuning method, referred to as 'down2_up2 method', was set as follows: (1) The optimizer was set to AdaGrad, and the learning rate was set to 0.005; (2) the learnable layers were those in 1st down, 2nd down, 1st up, and 2nd up blocks (down2_up2 model); and (3) the number of epochs was 20.

\subsection{Overall Workflow}

Figure 1 shows the overall workflow. First, we randomly divided the JC dataset into two halves; one was used for training and fine-tuning (JC pre-training dataset), whereas the other was used as an independent test dataset (JC independent test dataset). We balanced both training and test datasets according to the number of images obtained from each facility to optimize data distribution. Next, we created three types of machine learning models for segmentation. We created the BraTS model by training the algorithm using the BraTS dataset and the JC model using the JC pre-training dataset. We further established several fine-tuning models by fine-tuning the BraTS model using fewer than 20 cases from each facility among the JC pre-training dataset (Supplementary Figures S2-S4). The fine-tuning models were the BraTS models adjusted explicitly for each facility using only a limited amount of additional data. The number of the fine-tuning models was equal to the number of facilities. Finally, we evaluated each model's performance using the Dice coefficient score (see Materials and Methods Section 2.9) against our JC independent test dataset. 


\subsection{Performance Evaluation of Segmentation Models}

The performance of the segmentation models was evaluated by Dice coefficient scores. The Dice coefficient is a score that indicates the similarity between two samples. In the case of two images, the Dice coefficient score was calculated by dividing the number of pixels in the overlapping area times two by the number of pixels in both images. Therefore, the Dice coefficient score ranges from zero to one, and is one of the two images are an exact match.

\subsection{Statistical Analysis}

To randomly split the dataset, we used a random function from NumPy library (https:/ / docs.scipy.org/doc/numpy-1.14.0/reference/routines.random.html, accessed on 14 October 2019). To get robust results, we selected Welch's ANOVA to analyze variance and Games-Howell Post-Hoc Test as a post-hoc test. Welch's ANOVA and Games-Howell PostHoc Test do not assume equal variance and sample size [35,36]. We used oneway.test function from R core package (https:/ / www.rdocumentation.org/packages/stats/versions/3. 6.2/topics/oneway.test, accessed on 21 January 2020) and one-way function with GamesHowell option from R userfriendlyscience package (https:/ / www.rdocumentation.org/ packages/userfriendlyscience/versions/0.7.2/topics/oneway, accessed on 21 January 2020). With regard to the correlation analysis, we used the corrcoef function NumPy library for Python to compute the Pearson's correlation coefficient (https:/ / numpy.org/ $\mathrm{doc} /$ stable/reference/generated/numpy.corrcoef.html, accessed on 14 October 2019).

\section{Results}

3.1. Performance of Individual Models

\subsubsection{BraTS Model}

The Dice coefficient score of the BraTS model on the BraTS testing dataset was $0.873 \pm 0.098$ (Supplementary Figure S5); however, the score significantly decreased to $0.717 \pm 0.207$ when the BraTS model was applied to the JC independent test dataset. The results are shown in detail in Supplementary Figure S6. We observed the highest Dice coefficient score in the dataset from facility J $(0.817 \pm 0.065)$ and the lowest Dice coefficient score in the dataset from facility $\mathrm{G}(0.480 \pm 0.286)$.

\subsubsection{JC Model}

Next, we built and trained a JC model. The JC model's Dice coefficient score for the testing data of the JC dataset was $0.779 \pm 0.137$. As shown in Supplementary Figure S7, we observed the highest Dice coefficient score in facility $\mathrm{H}(0.836 \pm 0.051)$ and the lowest Dice coefficient score in facility $\mathrm{D}(0.673 \pm 0.273)$. The fluctuation in the Dice coefficient scores among the JC model facilities was observed to be smaller than that in the BraTS model (Supplementary Table S2).

\subsubsection{Fine-Tuning Models}

In fine-tuning models, the BraTS models were fine-tuned using a small set of data from the JC pre-training dataset. First, we searched for the best fine-tuning method using the JC pre-training dataset from facility A. Initially, we examined three different methods (Supplementary Figures S2-S4). As shown in Supplementary Figure S3, the 'fine all' model demonstrated the best performance, leading to the conclusion that both up path training and down path training were necessary to achieve optimal performance for fine-tuning.

Next, we investigated the effect of horizontal connection instead of the vertical connections of 3D U-Net on fine-tuning (Supplementary Figure S4). In this experiment, we re-examined three different methods (see method). As shown in Supplementary Figure S4, 'down2_up2 model' and 'fine_all model' showed comparable performances. In general, the fewer the parameters to be tuned, the lower the computational cost and the lesser the overfitting for the target domains with small data [37]; hence, 'down2_up2 model' was considered to be the most efficient fine-tuning model. 


\subsubsection{The Result of Fine-Tuning Models}

Using the down2_up2 method, we fine-tuned 10 BraTS models on the JC pre-training dataset from each facility. The mean Dice coefficient score of the fine-tuning models against each facility's JC pre-training dataset was $0.769 \pm 0.138$ (Supplementary Table S2).

\subsection{Comparison of the Three Models}

As shown in Figure 2, the Dice coefficient score of the fine-tuning model improved significantly compared with that of the BraTS model, and it was comparable to that of the JC model. In facilities B and J, the fine-tuning model's Dice coefficient score was higher than that of the JC model (Supplementary Table S2). Welch's ANOVA showed significant differences among the three groups $(p=0.0002)$. The Games-Howell Post-Hoc Test showed that there was a significant difference between the BraTS and JC models $(p<0.0001)$ and between the BraTS and fine-tuning models $(p=0.002)$ in terms of the Dice coefficient score. In contrast, there was no significant difference between the JC and fine-tuning models $(p=0.673)$.

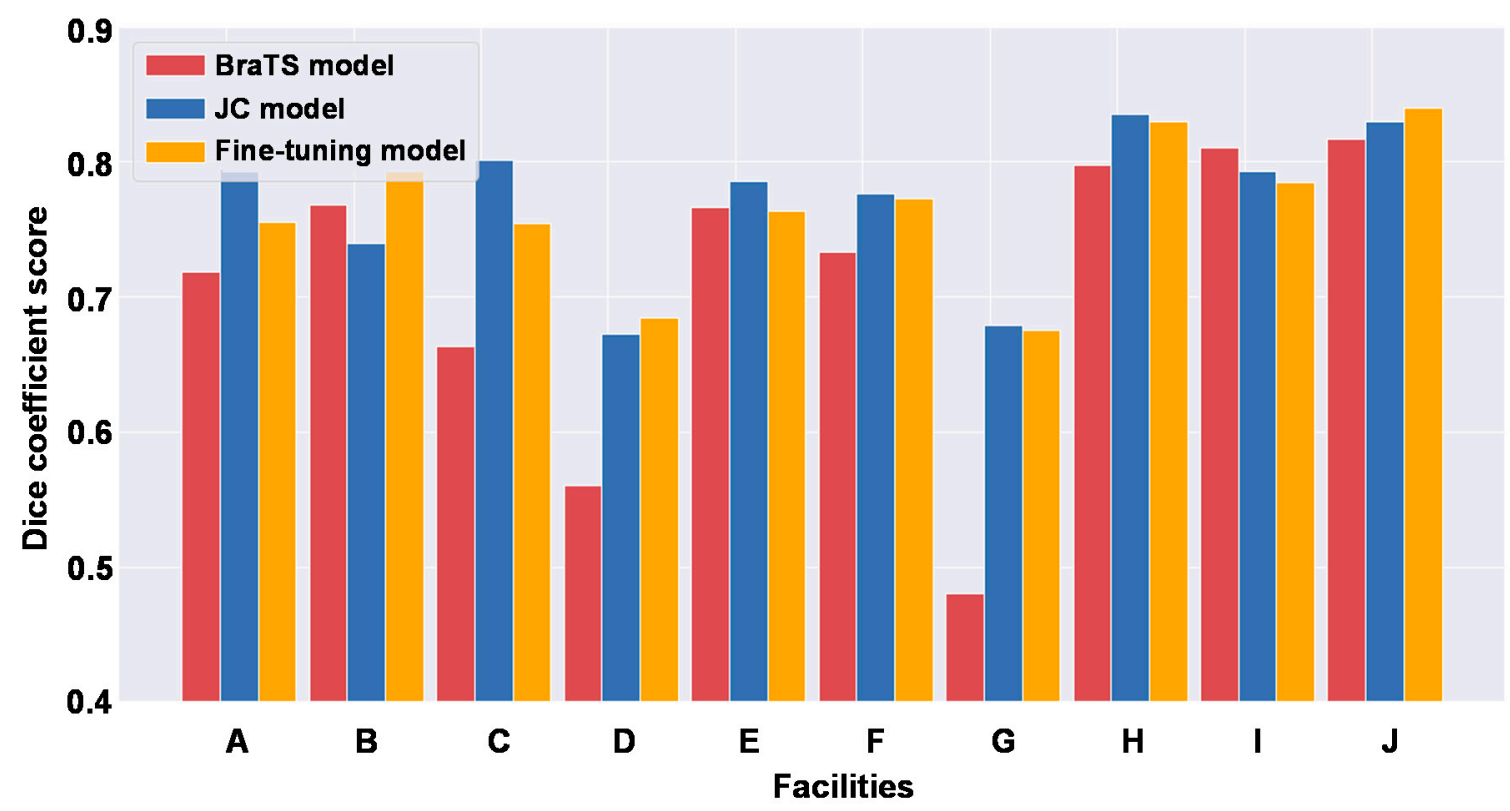

Figure 2. The results of three types of machine learning models for segmentation. A bar graph shows the Dice coefficient score for each facility. The horizontal axis is the facility, the vertical axis is the Dice coefficient score, and the colors indicate the types of machine learning models for segmentation. The Dice coefficient score of the fine-tuning model was significantly improved compared to that of the BraTS model and was comparable to that of the JC model.

Then, we focused on pathological diagnosis and comparison of three models. We show the results in Figure 3 and Supplementary Table S3. The Dice coefficient scores of the JC model and fine-tuning models tended to be better than those of the BraTS model, especially for oligodendroglioma and glioblastoma.

\subsection{Comparison of the VOI Obtained with the Three Models}

Figure 4 presents the segmentation results in representative cases from the testing data of the JC dataset. The VOI predicted by the BraTS model shows a larger area that includes normal brain tissue, not only the brain tumor. However, the fine-tuning model specific to the facility in Case 3 only shows the area that appears to be the tumor.

Here, we present three cases with the ground truth and predicted VOI. The three cases are cases from both datasets whose segmentation task seemed to be either very easy or difficult for the BraTS model. The first case, Case 1, is BraTS19_TCIA08_242_1 from testing data of the BraTS dataset (Supplementary Figure S8). Case 2 is from the testing data of the JC dataset (Supplementary Figure S9). The segmentation of these two cases seemed to be 
an easy task for machine learning models; the Dice coefficient score in the BraTS model was high (0.969 and 0.926). The final case is Case 3 from the testing data of the JC dataset, which is presented in Figure 4. The BraTS model could not predict the VOI accurately; the Dice coefficient score was low (0.330). Six histograms are presented to inspect the difference between the difficult and easy segmentations.

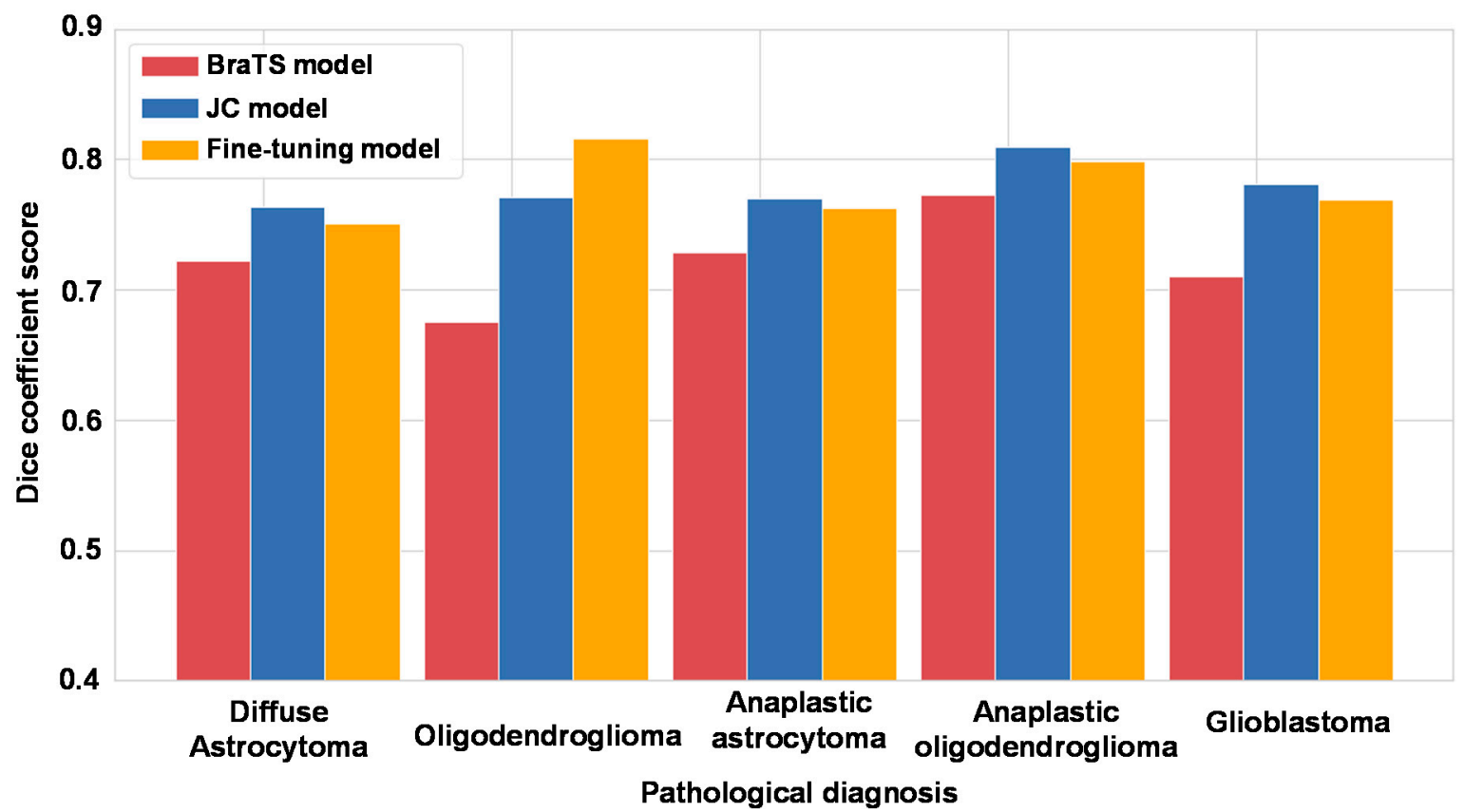

Figure 3. The results of three types of machine learning models for segmentation focused on pathological diagnosis. A bar graph shows the Dice coefficient score for each pathological diagnosis. The horizontal axis shows the pathological diagnosis, the vertical axis shows the Dice coefficient score, and the colors show the type of machine learning models for segmentation.
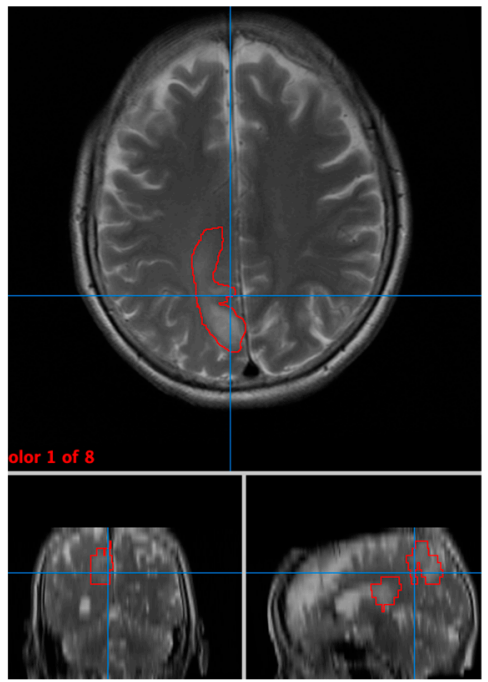

Ground truth

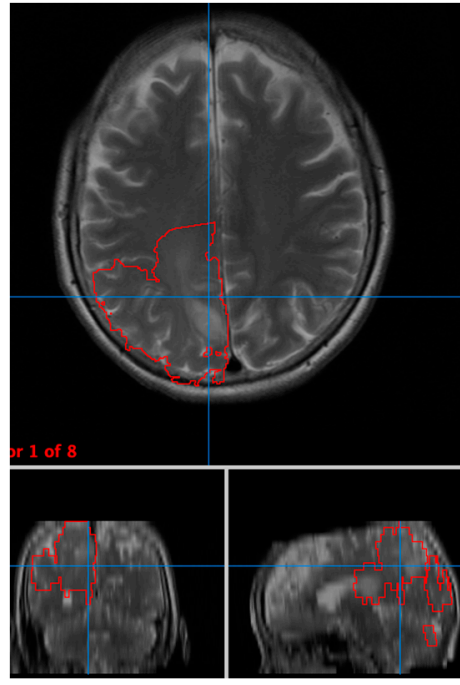

Prediction using BraTS model (Dice coefficient score 0.330 )

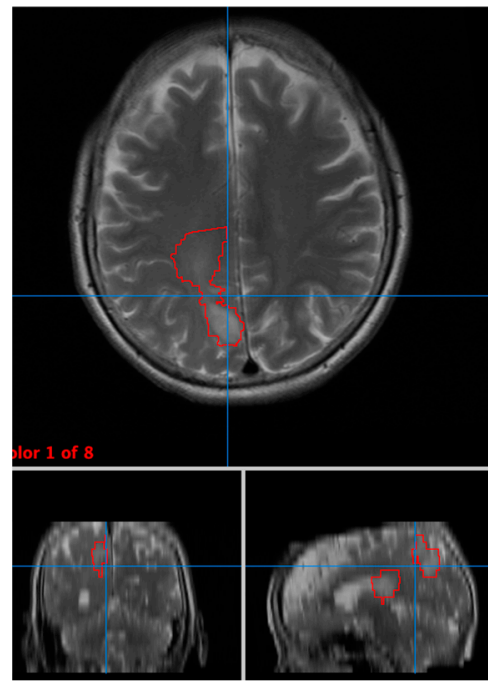

(Dice coefficient score 0.701)

Figure 4. Segmentation results on Case 3. The left column is ground truth (made by a skilled radiologist), the middle is predicted by the BraTS model, and the right is predicted by fine-tuning model specialized for the facility has Case 3 . The volume of interest (VOI) predicted by the BraTS model had an area that didn't seem to be a brain tumor. However, that by fine-tuning model specialized for the facility has Case 3 only have an area that seemed to be a tumor. 
In Figure 5A, we present the relationship between the four images. The GdT1 parts of the histograms of Case 1 and Case 2 have one peak around 1.8; however, Case 3 has no peak. Figure $5 \mathrm{~B}$ shows the relationship between the $\mathrm{T} 2$ image and true VOI as a histogram. The VOI part of the histograms of Case 1 and Case 2 is located at the right edge (around 3) and occupies a relatively large portion. However, that of Case 3 is located centrally (around 2.5) and covers a small portion.

A

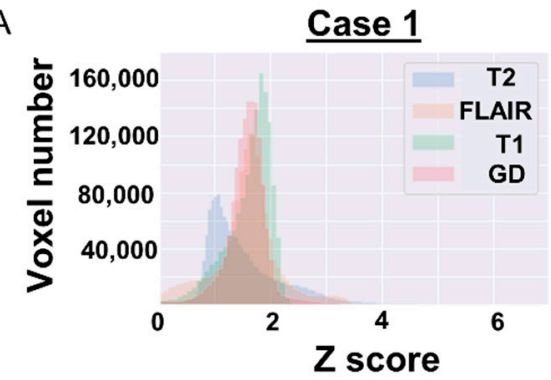

B

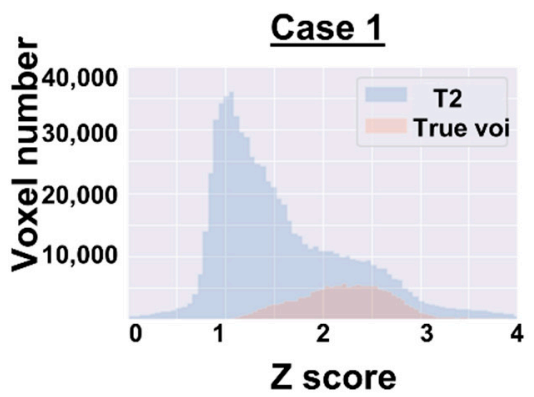

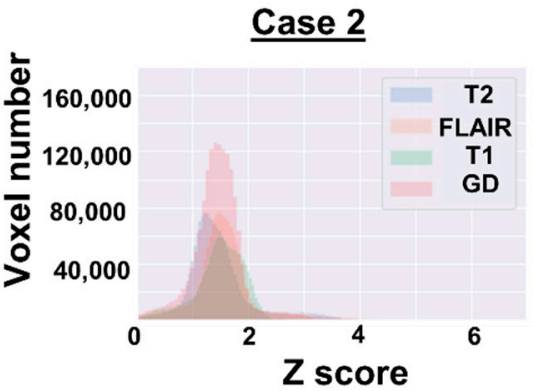

Case 2

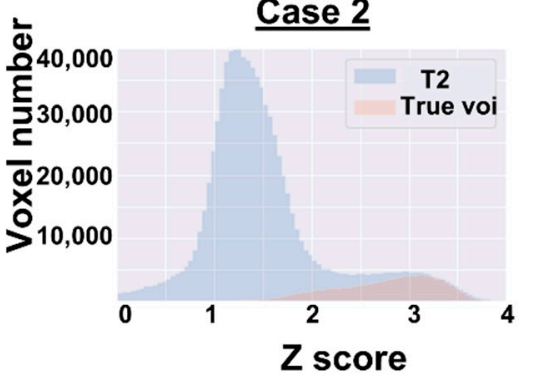

Case 3

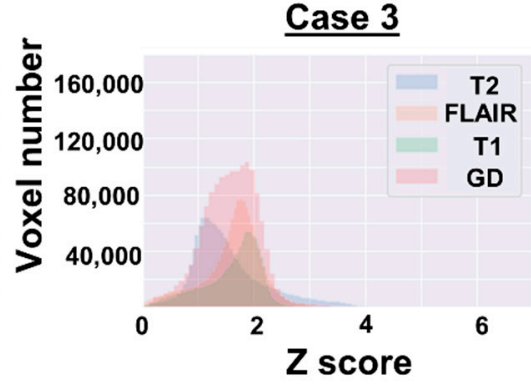

Case 3

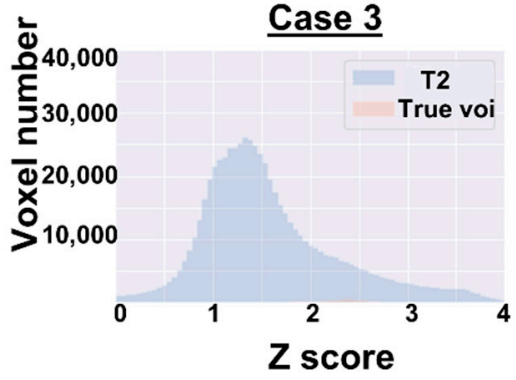

Figure 5. The results of comparison of image types in Case 1 from the BraTS dataset and Case 2 and Case 3 cases from the JC dataset are shown in histograms. The horizontal axis of the histogram represents the voxel value converted into a Z-score, and the vertical axis represents the number of voxels. (A) Each color represents image types. The GdT1 part of histograms of Case 1 and Case 2 have one peak around 1.8, however that of Case 3 has no peak. (B) The image histograms show the relationship between the T2 image (equivalent to the slice corresponding to the VOI) and true VOI. Blue histogram represents the T2 image, and orange represents true VOI. The VOI part of histograms of Case 1 and Case 2, located right edge (around 3), occupied a relatively large portion. But that of Case 3 was located central (around 2.5) and had a small portion.

The tumor volume of Case 3 was small $(12.9 \mathrm{~mL})$. Then, further analysis was performed to study the correlation between the Dice coefficient and tumor volume. Supplementary Figure S10 is a scatter plot of the Dice coefficient score and tumor volume. When the correlation coefficient was calculated using Pearson product-moment correlation coefficient, there was no correlation between the Dice coefficient and tumor volume for all three model types. However, the correlation coefficient of the BraTS model was slightly higher than that of the JC model and the fine-tuning model (0.246 [BraTS model], 0.152 [JC model], and 0.156 [fine-tuning model]). In addition, when we focused on the cases with the Dice coefficient lower than $0.6,60 \%(33 / 55)$ of the cases had a tumor volume of less than $50 \mathrm{~mL}$. Judging from this fact, the trend that when the tumor volume is small (less than $50 \mathrm{~mL}$ ), the Dice coefficient is also low (less than 0.6) was observed in all three model types.

\section{Discussion}

In the present study, we demonstrated that the significant performance degradation of a machine learning model for glioma segmentation was caused by the domain shift (Supplementary Figures S5 and S6). Furthermore, we observed significant variation in the performance degradation among facilities. We believe that the fine-tuning method developed in this study would be a useful way to solve this issue (Figures 2 and 4, and Supplementary Figure S11).

Because of glioma's invasive nature, it is difficult to determine the precise boundary between tumors and normal brain tissues in MR images. However, determining the precise 
boundary is considerably important for both high- and low-grade gliomas before the surgery because more extensive resection of the tumor is associated with better outcomes [8] To prevent postoperative neurological dysfunction, preoperative recognition of neural anatomy and clear tumor boundary is needed.

In addition, it is also important to determine the boundary of gliomas accurately when evaluating the effect of the treatment in clinical trials based on response assessment criteria in neuro-oncology. Various clinical trials for gliomas are ongoing, and therapeutic effects are largely assessed by the radiological response of MR images. In multi-institutional clinical trials, the boundary must be created based on the same criteria, but it can differ among clinicians who evaluate it. Overall, the situation that we need to determine the precise boundary between a tumor and normal brain tissues in MR images has frequently occurred in clinical settings. Therefore, robust machine learning models for tumor segmentation are required.

The determination of the boundary between a tumor and normal brain tissues in gliomas relies on the abilities of skilled radiologists. For this reason, it is conceivable that a machine learning model for glioma segmentation is particularly useful for facilities where there are no skilled radiologists. The number of glioma cases in facilities where no skilled neuroradiologists are working is generally low. Given that numerous MR images are required to build a machine learning model for glioma segmentation, it would be unrealistic if a model has to be built from the beginning in each facility. Our fine-tuning method can offer the solution because it requires fewer than 20 glioma cases.

Although many researchers recognize that domain shifts reduce the performance of machine learning models, this problem has been poorly investigated [38-40]. One of the main reasons for poor investigation is the requirement of datasets from multiple facilities. In this regard, Pooch et al. recently reported that poor performance of models, due to the domain shift, was observed even in chest radiographs, which have less information and diversity among facilities than 3D brain volumes [41]. AlBadawy et al. also reported the poor performance of models caused by the domain shift in MR images of gliomas from two different facilities in The Cancer Imaging Archive (TCIA) public dataset [42]. This result is noteworthy because MR images of gliomas in the TCIA seem to be more homogeneous than those in the JC dataset. Homogeneity is an advantage when analyzing within the same dataset, but a disadvantage when extrapolating to other datasets.

Our JC dataset was collected from 10 facilities during an extremely long period of time from 1991 to 2015. We visualized the diversity of image resolutions of the JC dataset using the size of voxels (Supplementary Figure S12 and Table S4). For example, the voxel size in the $z$-axis, which corresponds to the slice thickness, varied widely from 3.3 to $9 \mathrm{~mm}$. MR scanners and image settings also varied. This fact indicates that the JC dataset is heterogeneous and likely represents real-world data.

We used the JC dataset and found highly frequent and severe performance degradation of a machine learning model caused by the domain shift. It should be noted that the severity of performance degradation varied substantially among domains (Figure 2 and Supplementary Figure S6). This result implies that it is difficult to guarantee the performance of a machine learning model, which is trained only on a public dataset, such as the BraTS dataset.

Although the network architecture of U-Net is frequently used when building machine learning models for segmentation, it has rarely been investigated for fine-tuning. Amiri et al. recently assessed the effects of fine-tuning different layers of the U-Net model that was trained on 1000 segmented natural images for segmentation of breast ultrasound images [39], and concluded that setting the shallow layer as a learnable layer was more effective than setting the deep layer. They also mentioned that concordant results were not observed in the segmentation of radiographs.

In this study, we demonstrated that the most effective fine-tuning model is the down2_up2 model (learnable layers were those in 1st down, 2nd down, 1st_up, and 2nd_down blocks, Supplementary Figures S2 and S4). The architecture of U-Net is divided 
into two phases; the first phase performs a convolution to obtain a latent representation, and the other phase reconstructs an image from the latent representation. The phase of obtaining a latent representation of U-Net is almost the same as that of a classic convolutional neural network $(\mathrm{CNN})$. The $\mathrm{CNN}$ representations of shallow layers are lower-level features (e.g., lines, edges, and blobs), and the CNN representations of deep layers are more semantic and higher-level features. In our experience, the image differences among facilities seem to be caused by the diversity of instruments and methods in each facility. We assume that the image differences among facilities could be processed by low-level features, corresponding to shallow layers in $\mathrm{CNN}$. Hence, it is reasonable to make layer groups that consist of a shallow layer block and a deep layer block, which is directly connected to the shallow layer. Our results are compatible with the results obtained by Amiri et al. [39].

As shown in Figure 5A, the shapes of the histograms of Case 1 and Case 2 are similar; however, that of Case 3 is slightly different. The differences in image quality cannot be normalized even after the Z-score conversion might have caused this histogram difference. Therefore, in Case 1 and Case 2, the VOIs account for a large percentage of the histogram and are located at the right tail. In other words, the tumors of Case 1 and Case 2 are large, and there are a few voxels having a higher voxel value than that of the part of the tumor. Segmentation of such cases is considered to be an easy task for the machine learning model. In Case 3, the region occupied by the VOI is small, and the VOI exists close to the center of the histogram. Segmentation of such cases appears to be difficult for the machine learning model. As shown in Figure 4, our fine-tuning method seems to work in Case 3 and could make the VOI close to the ground truth. Because a relatively small number of cases (fewer than 20 cases) are sufficient to show good performance, our method is useful when creating a machine learning model in a facility where there are a small number of glioma cases. Since we believe that all patients with gliomas should ideally be treated at a complex oncological facility, we hope that our machine learning method will help local hospitals, where such patients may visit for the first time, to appropriately direct patients to referral facilities.

As shown in Table 1, the data obtained from a single facility are always biased. To deploy a high-performance machine learning model, it is ideal to obtain unbiased data, such as the data from one large domain; however, this is rarely realized. Therefore, there may be two things that are required for building an accurate machine learning model in a clinical setting. First, it is important to collect as many images as possible from multiple facilities for building a model. Second, we must optimize the model corresponding to the 'data bias' of the facility. In our view, turning the two steps into a cycle can gradually create a 'truly useful' machine learning model in clinical sites.

Our study has some limitations. Firstly, our fine-tuning method works for our 3D U-Net model, but may not always work for other improved U-Net models. Furthermore, our fine-tuning method is likely to be useful in glioma segmentation; however, it is unclear whether it will work for other types of brain tumors, including brain metastases or primary central nervous system lymphoma. Further verification may clarify this point. The JC dataset is very large and heterogeneous as a glioma dataset; nonetheless, verification with another large dataset is needed in the future.

\section{Conclusions}

Our study explored the performance degradation of the machine learning model for glioma segmentation caused by the domain shift; the fine-tuning method developed in this study can be a useful way to solve this issue. Because our fine-tuning method only requires fewer than 20 cases, this method is useful when building a machine learning model for segmentation in a facility where the number of glioma cases is small.

Supplementary Materials: The following are available online at https:/ / www.mdpi.com/2072-669 4/13/6/1415/s1, Figure S1: Segmentation results on Case 2 from the testing data of the JC dataset. The left column is ground truth (made by a skilled radiologist), right is predicted by the BraTS model, Figure S2: The architecture of machine learning model for segmentation, Figure S3: The performances 
of "fine_all" model, "up" model, and "down" model, Figure S4: The performances of "fine_all" model, "down1_up1" model, and "down2_up2" model, Figure S5: The Dice coefficient scores of the BraTS models for the testing data of the BraTS dataset, Figure S6: The Dice coefficient scores of the BraTS model for the testing data of the JC dataset, Figure S7: The Dice coefficient scores of the JC model for the testing data of the JC dataset, Figure S8: Segmentation results on Case 1 from testing data of BraTS dataset. The left column is ground truth, right is predicted by the BraTS model, Figure S9: Segmentation results on Case 2 from testing data of the JC dataset. The left column is ground truth, right is predicted by the BraTS model, Figure S10: A scatter plots about Dice coefficient score and tumor volumes, Figure S11: The Dice coefficient scores of the fine-tuning models for the testing data of the JC dataset, Figure S12: Summary of voxel sizes of images of the JC dataset, Table S1: Summary of our 3D U-Net architecture, Table S2: Comparison of the Dice coefficient score for the testing data of the JC dataset, Table S3: Comparison of the Dice coefficient score for the testing data of the JC dataset focus on pathological diagnosis, Table S4: Summary of voxel sizes of the JC dataset. Each column represents the type of image sequences and axis. For example, 'FLAIR_x' column summarizes $x$ axis voxel size of FLAIR (fluid-attenuated inversion recovery).

Author Contributions: Conceptualization, S.T. (Satoshi Takahashi), M.T., M.K., R.H.; methodology, Satoshi Takahashi, M.M., M.K, R.K., K.K., J.S.; formal analysis, Satoshi Takahashi, R.K.; resources, M.T., Y.N., N.S., A.M., K.S., M.N. (Motoo Nagane), R.O., F.H., S.T. (Shota Tanaka), N.H., K.T. (Kaoru Tamura), K.T. (Kensuke Tateishi), R.N., H.A., M.N. (Masahiro Nonaka), T.U., J.F., Y.O., N.T., Y.K.; data curation, M.T., M.K., M.M.; writing—original draft preparation, Satoshi Takahashi, M.T., M.K., J.S., R.H.; writing-review and editing, visualization. M.T., M.K., Motoo Nagane, J.S., R.H.; supervision, K.I., Y.N., R.H. All authors have discussed on the concept of the study. All authors have read and agreed to the published version of the manuscript.

Funding: This work was supported by JST CREST [Grant Number JPMJCR1689] and JSPS Grant-inAid for Scientific Research on Innovative Areas [Grant Number JP18H04908].

Institutional Review Board Statement: The study was conducted according to the guidelines of the Declaration of Helsinki, and approved by the Institutional Review Board of National Cancer Center (protocol code: 2013-042 and 2016-496, approved date: 10 May 2013 and 28 June 2017, respectively).

Informed Consent Statement: Informed consent was obtained from all subjects for the treatment and anonymous clinical data is allowed to use in a retrospective study. Also, we applied opt-out method to obtain consent on this study that was approved by the Institutional Review Board of National Cancer Center.

Data Availability Statement: The data presented in this study are available in this article (and Supplementary Material).

Acknowledgments: We would like to thank all members of R. Hamamoto's Lab. They provided us valuable advice and comfort environment. We also would like to thank Toshimitsu Utsuno, Tatsuya Horita, Yuji Jibiki, Keisuke Ueki, Taishi Nakamura, Hideo Nakamura, Junichi Adachi, Koji Yoshimoto, and Noriyuki Kijima for their dedicated works for the preparation of the image dataset.

Conflicts of Interest: No potential conflicts of interest were disclosed by any author in this study.

Meeting Presentation: Some part of this study was presented at 25th Society for Neuro-Oncology meeting as an abstract number of NIMG-29.

\section{References}

1. Rasmussen, B.K.; Hansen, S.; Laursen, R.J.; Kosteljanetz, M.; Schultz, H.; Nørgård, B.M.; Guldberg, R.; Gradel, K.O. Epidemiology of glioma: Clinical characteristics, symptoms, and predictors of glioma patients grade I-IV in the the Danish Neuro-Oncology Registry. J. Neuro-Oncology 2017, 135, 571-579. [CrossRef] [PubMed]

2. Arita, H.; Narita, Y.; Fukushima, S.; Tateishi, K.; Matsushita, Y.; Yoshida, A.; Miyakita, Y.; Ohno, M.; Collins, V.P.; Kawahara, N.; et al. Upregulating mutations in the TERT promoter commonly occur in adult malignant gliomas and are strongly associated with total 1p19q loss. Acta Neuropathol. 2013, 126, 267-276. [CrossRef] [PubMed]

3. Ichimura, K.; Nishikawa, R.; Matsutani, M. Molecular markers in pediatric neuro-oncology. Neuro-Oncology 2012, 14, iv90-iv99. [CrossRef] [PubMed]

4. Hegi, M.E.; Diserens, A.-C.; Gorlia, T.; Hamou, M.-F.; De Tribolet, N.; Weller, M.; Kros, J.M.; Hainfellner, J.A.; Mason, W.; Mariani, L.; et al. MGMTGene Silencing and Benefit from Temozolomide in Glioblastoma. N. Engl. J. Med. 2005, 352, 997-1003. [CrossRef] [PubMed] 
5. $\quad$ Lassman, A.B.; Iwamoto, F.M.; Cloughesy, T.F.; Aldape, K.D.; Rivera, A.L.; Eichler, A.F.; Louis, D.N.; Paleologos, N.A.; Fisher, B.J.; Ashby, L.S.; et al. International retrospective study of over 1000 adults with anaplastic oligodendroglial tumors. Neuro-Oncology 2011, 13, 649-659. [CrossRef]

6. Penas-Prado, M.; De Groot, J. CATNON interim results: Another triumph of upfront chemotherapy in glioma. Neuro-Oncology 2017, 19, 1287-1288. [CrossRef]

7. Stupp, R.; Mason, W.P.; van den Bent, M.J.; Weller, M.; Fisher, B.; Taphoorn, M.J.B.; Belanger, K.; Brandes, A.A.; Marosi, C.; Bogdahn, U.; et al. Radiotherapy plus Concomitant and Adjuvant Temozolomide for Glioblastoma. N. Engl. J. Med. 2005, 352, 987-996. [CrossRef]

8. Sanai, N.; Berger, M.S. Glioma extent of resection and its impact on patient outcome. Neurosurg. 2008, 62, 753-766. [CrossRef]

9. Ciçek, Ö.; Abdulkadir, A.; Lienkamp, S.S.; Brox, T.; Ronneberger, O. 3D U-Net: Learning Dense Volumetric Segmentation from Sparse Annotation. In Medical Image Computing and Computer-Assisted Intervention-MICCAI 2016. MICCAI 2016. Lecture Notes in Computer Science; Ourselin, S., Joskowicz, L., Sabuncu, M., Unal, G., Wells, W., Eds.; Springer: Cham, Switzerland, 2016; Volume 9901. [CrossRef]

10. Akkus, Z.; Galimzianova, A.; Hoogi, A.; Rubin, D.L.; Erickson, B.J. Deep Learning for Brain MRI Segmentation: State of the Art and Future Directions. J. Digit. Imaging 2017, 30, 449-459. [CrossRef]

11. Havaei, M.; Davy, A.; Warde-Farley, D.; Biard, A.; Courville, A.; Bengio, Y.; Pal, C.; Jodoin, P.-M.; Larochelle, H. Brain tumor segmentation with Deep Neural Networks. Med. Image Anal. 2017, 35, 18-31. [CrossRef]

12. Kamnitsas, K.; Ledig, C.; Newcombe, V.F.; Simpson, J.P.; Kane, A.D.; Menon, D.K.; Rueckert, D.; Glocker, B. Efficient multi-scale 3D CNN with fully connected CRF for accurate brain lesion segmentation. Med. Image Anal. 2017, 36, 61-78. [CrossRef]

13. Pereira, S.; Pinto, A.; Alves, V.; Silva, C.A. Brain Tumor Segmentation Using Convolutional Neural Networks in MRI Images. IEEE Trans. Med. Imaging 2016, 35, 1240-1251. [CrossRef] [PubMed]

14. Liu, Z. Automatic Segmentation of Non-Tumor Tissues in Glioma MR Brain Images Using Deformable Registration with Partial Convolutional Networks. arXiv 2020, in press.

15. Stawiaski, J. A Multiscale Patch Based Convolutional Network for Brain Tumor Segmentation. arXiv 2017, in press.

16. Menze, B.H.; Jakab, A.; Bauer, S.; Kalpathy-Cramer, J.; Farahani, K.; Kirby, J.; Burren, Y.; Porz, N.; Slotboom, J.; Wiest, R.; et al. The Multimodal Brain Tumor Image Segmentation Benchmark (BRATS). IEEE Trans. Med. Imaging 2015, 34, 1993-2024. [CrossRef]

17. Myronenko, A. 3D MRI Brain Tumor Segmentation Using Autoencoder Regularization. In International MICCAI Brainlesion Workshop; Springer: Cham, Switzerland, 2018; pp. 311-320.

18. Komatsu, M.; Sakai, A.; Komatsu, R.; Matsuoka, R.; Yasutomi, S.; Shozu, K.; Dozen, A.; Machino, H.; Hidaka, H.; Arakaki, T.; et al. Detection of Cardiac Structural Abnormalities in Fetal Ultrasound Videos Using Deep Learning. Appl. Sci. 2021, 11, 371. [CrossRef]

19. Asada, K.; Kobayashi, K.; Joutard, S.; Tubaki, M.; Takahashi, S.; Takasawa, K.; Komatsu, M.; Kaneko, S.; Sese, J.; Hamamoto, R. Uncovering Prognosis-Related Genes and Pathways by Multi-Omics Analysis in Lung Cancer. Biomolecules 2020, $10,524$. [CrossRef]

20. Hamamoto, R.; Komatsu, M.; Takasawa, K.; Asada, K.; Kaneko, S. Epigenetics Analysis and Integrated Analysis of Multiomics Data, Including Epigenetic Data, Using Artificial Intelligence in the Era of Precision Medicine. Biomolecules 2019, 10, 62. [CrossRef]

21. Hamamoto, R.; Suvarna, K.; Yamada, M.; Kobayashi, K.; Shinkai, N.; Miyake, M.; Takahashi, M.; Jinnai, S.; Shimoyama, R.; Sakai, A.; et al. Application of Artificial Intelligence Technology in Oncology: Towards the Establishment of Precision Medicine. Cancers 2020, 12, 3532. [CrossRef]

22. Jinnai, S.; Yamazaki, N.; Hirano, Y.; Sugawara, Y.; Ohe, Y.; Hamamoto, R. The Development of a Skin Cancer Classification System for Pigmented Skin Lesions Using Deep Learning. Biomolecules 2020, 10, 1123. [CrossRef]

23. Dozen, A.; Komatsu, M.; Sakai, A.; Komatsu, R.; Shozu, K.; Machino, H.; Yasutomi, S.; Arakaki, T.; Asada, K.; Kaneko, S.; et al. Image Segmentation of the Ventricular Septum in Fetal Cardiac Ultrasound Videos Based on Deep Learning Using Time-Series Information. Biomolecules 2020, 10, 1526. [CrossRef] [PubMed]

24. Shozu, K.; Komatsu, M.; Sakai, A.; Komatsu, R.; Dozen, A.; Machino, H.; Yasutomi, S.; Arakaki, T.; Asada, K.; Kaneko, S.; et al. Model-Agnostic Method for Thoracic Wall Segmentation in Fetal Ultrasound Videos. Biomolecules 2020, 10, 1691. [CrossRef] [PubMed]

25. Yamada, M.; Saito, Y.; Imaoka, H.; Saiko, M.; Yamada, S.; Kondo, H.; Takamaru, H.; Sakamoto, T.; Sese, J.; Kuchiba, A.; et al. Development of a real-time endoscopic image diagnosis support system using deep learning technology in colonoscopy. Sci. Rep. 2019, 9, 1-9. [CrossRef] [PubMed]

26. Yasutomi, S.; Arakaki, T.; Matsuoka, R.; Sakai, A.; Komatsu, R.; Shozu, K.; Dozen, A.; Machino, H.; Asada, K.; Kaneko, S.; et al. Shadow Estimation for Ultrasound Images Using Auto-Encoding Structures and Synthetic Shadows. Appl. Sci. 2021, $11,1127$. [CrossRef]

27. Torralba, A.; Efros, A.A. Unbiased look at dataset bias. CVPR 2011 2011, 10, 1521-1528. [CrossRef]

28. LeCun, Y.; Bengio, Y.; Hinton, G. Deep learning. Nature 2015, 521, 436-444. [CrossRef] [PubMed]

29. Arita, H.; Yamasaki, K.; Matsushita, Y.; Nakamura, T.; Shimokawa, A.; Takami, H.; Tanaka, S.; Mukasa, A.; Shirahata, M.; Shimizu, S.; et al. A combination of TERT promoter mutation and MGMT methylation status predicts clinically relevant subgroups of newly diagnosed glioblastomas. Acta Neuropathol. Commun. 2016, 4, 1-14. [CrossRef] 
30. Bakas, S.; Akbari, H.; Sotiras, A.; Bilello, M.; Rozycki, M.; Kirby, J.S.; Freymann, J.B.; Farahani, K.; Davatzikos, C. Advancing The Cancer Genome Atlas glioma MRI collections with expert segmentation labels and radiomic features. Sci. Data 2017, 4, 170117. [CrossRef]

31. Pei, L.; Vidyaratne, L.; Rahman, M.; Iftekharuddin, K.M. Context aware deep learning for brain tumor segmentation, subtype classification, and survival prediction using radiology images. Sci. Rep. 2020, 10, 1-11. [CrossRef]

32. Smith, S.M. Fast robust automated brain extraction. Hum. Brain Mapp. 2002, 17, 143-155. [CrossRef]

33. Isensee, F.; Kickingereder, P.; Wick, W.; Bendszus, M.; Maier-Hein, K.H. Brain Tumor Segmentation and Radiomics Survival Prediction: Contribution to the BRATS 2017 Challenge; Lecture Notes in Computer Science Series; Springer: Cham, Switzerland, 2018; pp. 287-297.

34. Kayalibay, B.; Jensen, G.; van der Smagt, P. CNN-based segmentation of medical imaging data. arXiv 2017, in press.

35. Ruxton, G.D. The unequal variance t-test is an underused alternative to Student's t-test and the Mann-Whitney U test. Behav. Ecol. 2006, 17, 688-690. [CrossRef]

36. Ruxton, G.D.; Beauchamp, G. Time for some a priori thinking about post hoc testing. Behav. Ecol. 2008, 19, 690-693. [CrossRef]

37. Yosinski, J.; Clune, J.; Bengio, Y.; Lipson, H. How Transferable Are Features in Deep Neural Networks? arXiv 2014, in press.

38. Madani, A.; Moradi, M.; Karargyris, A.; Syeda-Mahmood, T. Semi-supervised learning with generative adversarial networks for chest X-ray classification with ability of data domain adaptation. In Proceedings of the 2018 IEEE 15th International Symposium on Biomedical Imaging (ISBI 2018), Washington, DC, USA, 4-7 April 2018; Institute of Electrical and Electronics Engineers (IEEE): Piscataway, NJ, USA, 2018; pp. 1038-1042.

39. Amiri, M.; Brooks, R.; Rivaz, H. Fine Tuning U-Net for Ultrasound Image Segmentation: Which Layers? Springer International Publishing: Cham, Switzerland, 2019; pp. 235-242.

40. Chen, C.; Dou, Q.; Chen, H.; Heng, P.-A. Semantic-Aware Generative Adversarial Nets for Unsupervised Domain Adaptation in Chest $X$-Ray Segmentation; Lecture Notes in Computer Science Series; Springer: Cham, Switzerland, 2018; pp. $143-151$.

41. Pooch, E.H.P.; Ballester, P.L.; Barros, R.C. Can we trust deep learning models diagnosis? The impact of domain shift in chest radiograph classification. arXiv 2020, in press.

42. Albadawy, E.A.; Saha, A.; Mazurowski, M.A. Deep learning for segmentation of brain tumors: Impact of cross-institutional training and testing. Med. Phys. 2018, 45, 1150-1158. [CrossRef] 